\title{
Fair Scheduling With QoS Support in Wireless Ad Hoc Networks
}

\author{
Hsi-Lu Chao, Student Member, IEEE and Wanjiun Liao, Member, IEEE
}

\begin{abstract}
In this paper, we study fair scheduling with quality of service (QoS) support for wireless ad hoc networks. Two types of flows are considered: best effort and guaranteed flows. The goal is to satisfy the minimum bandwidth requirements of guaranteed flows and to provide a fair share of residual bandwidth to all flows. We compare timestamp-based and credit-based mechanisms, and evaluate the feasibility of all existing fair scheduling schemes of these two types of mechanisms for multimedia wireless multihop networks. We suggest a flow weight calculation scheme for existing timestamp-based mechanisms to support both best effort and guaranteed flows, and propose a credit-based mechanism called credit-based slot allocation protocol (CSAP) for fair scheduling with QoS support. For comparison purposes, several metrics are defined to evaluate the performances of these two kinds of mechanisms. The simulation results show that CSAP outperforms the other approaches in terms of meeting the minimum requirements of guaranteed flows, fairly sharing the residual bandwidth among all flows, and improving overall system throughput.
\end{abstract}

Index Terms-Ad hoc networks, fair scheduling, quality of service (QoS).

\section{INTRODUCTION}

A $\mathrm{N}$ ad hoc network is a self-organizing wireless network composed only of mobile nodes without the support of any preexisting wired infrastructure. In such a network, each node plays both roles of a terminal and a router. Due to the fully distributed characteristic of the network, collisions may occur and the system throughput may be low without proper coordination among the transmitting nodes.

Providing quality of service (QoS) in ad hoc networks is a new area of research. Existing work focuses mainly on QoS routing [1], [2], which finds a path to meet the desired service levels of flows. This paper studies a different QoS issue in multihop, multimedia wireless networks. We consider a mix of guaranteed and best effort flows and investigate fair scheduling with QoS support for the network. The goal is to guarantee the minimum bandwidth requirements of guaranteed flows and to ensure a fair share of residual bandwidth for all flows. In the following, we first define "fairness" in the context of ad hoc networks, and then examine related issues of fair scheduling with QoS support for multimedia ad hoc networks.

Manuscript received August 8, 2002; revised July 9, 2003; accepted September 2, 2003. The editor coordinating the review of this paper and approving it for publication is $\mathrm{B}$. Li. This work was supported in part by the MOE program for Promoting Academic Excellence of Universities under Grant 89E-FA06-2-4-7 and in part by the National Science Council, Taiwan, under Grant NSC91-2213-E-002-057.

The authors are with the Department of Electrical Engineering and Graduate Institute of Communication Engineering, National Taiwan University, Taipei 106, Taiwan, R.O.C. (e-mail: wjliao@ cc.ee.ntu.edu.tw).

Digital Object Identifier 10.1109/TWC.2004.837269

\section{A. Fairness}

Fairness is an important criterion of resource sharing in the best effort Internet, especially when there is competition for their share due to unsatisfied demands [3]. Fairness is defined as follows [4]-[6]. Each flow $f$ is allowed to share a certain percentage of link capacity based on its flow weight indicated as $w_{f}$. Let $W_{f}\left(t_{1}, t_{2}\right)$ denote the aggregate resource received by flow $f$ in time interval $\left[t_{1}, t_{2}\right]$. The allocation is called ideal fair if (1) is satisfied for all intervals $\left[t_{1}, t_{2}\right]$ in which both flows $f$ and $g$ are backlogged

$$
\left|\frac{W_{f}\left(t_{1}, t_{2}\right)}{w_{f}}-\frac{W_{g}\left(t_{1}, t_{2}\right)}{w_{g}}\right|=0 .
$$

Scheduling is an effective way to ensure fair sharing of resource among flows [7]. It determines the service order of packets according to such metrics as packet timestamp, delay bound, and cumulative credit. Of these metrics, timestamp is the most common one used by existing scheduling schemes [8]-[11]. For these timestamp-based scheduling protocols, the system maintains a virtual time as in [4]. Each packet is associated with two timestamps, i.e., start tag and finish tag, based on the system-defined virtual time. Let $S_{f}^{j}$ and $F_{f}^{j}$ denote the start tag and the finish tag for the $j$ th packet of flow $f$, respectively

$$
\left\{\begin{array}{l}
S_{f}^{j}=\max \left\{v\left[A\left(p_{f}^{j}\right)\right], F_{f}^{j-1}\right\}, \quad j \geq 1 \\
F_{f}^{j}=S_{f}^{j}+\frac{l_{f}^{j}}{w_{f}}, \quad j \geq 1
\end{array}\right.
$$

where $p_{f}^{j}, l_{f}^{j}$ and $A\left(p_{f}^{j}\right)$ are the $j$ th packet of flow $f$, the length of $p_{f}^{j}$, and the arrival time of $p_{f}^{j}$, respectively. $v\left[A\left(p_{f}^{j}\right)\right]$ denotes the virtual arrival time of $p_{f}^{j}$.

The main objective of existing fair scheduling approaches is to ensure $E$, defined in (3), as close to zero as possible. The closer $E$ is to zero, the fairer the approach

$$
E=\left|\frac{W_{f}\left(t_{1}, t_{2}\right)}{w_{f}}-\frac{W_{g}\left(t_{1}, t_{2}\right)}{w_{g}}\right| .
$$

\section{B. Design Issues of Fair Scheduling in Ad Hoc Networks}

Due to the characteristics of wireless ad hoc networks, three challenges must be addressed when a fair scheduling protocol is designed [9].

1) Location-Dependent Contention: Wireless transmission is broadcast locally to the network. Uncoordinated simultaneous transmissions may result in packet collisions. Those flows that incur packet collisions when transmitted simultaneously are called contending flows. For example, in Fig. 1, there are nine nodes (denoted N1, N2, .., N9) and four flows (denoted F1, 
F2, F3, F4). The line connecting two nodes indicates each is within the transmission range of the other. Flows F1 and F2 cannot be transmitted simultaneously, or a collision occurs at $\mathrm{N} 2$. Node N4 is interfered by flow F3, because node N4 is within the transmission range of node N7. Likewise, nodes N3 and N5 are interfered by flow F4.

2) Spatial Channel Reuse: Multiple flows can be transmitted simultaneously without collisions if these flows do not interfere with each other. This is called spatial channel reuse. For example, flows F2 and F3 in Fig. 1 can be transmitted simultaneously. Spatial channel reuse is an important technique to improve system throughput. A good design of fair scheduling should provide a simple and cost-effective way of finding the maximum number of flows that can be transmitted simultaneously.

3) Conflict Between Global Fairness and Maximal Channel Utilization for Wireless Systems: Existing work on fair scheduling for ad hoc networks focuses on global fairness, because all flows are assumed in a single cluster. Typically, a maximum window size is defined to restrict the maximum bandwidth each flow can use in a time interval. In reality, wireless networks, such as IEEE 802.11 and Bluetooth, are cluster-based and make use of channel reuse to maximize system throughput. A good mechanism should balance the tradeoff between fairness and maximum channel reuse when fair scheduling is provided.

\section{Related Work}

1) Fair Scheduling in Ad Hoc Networks: Most existing works for fair scheduling in ad hoc networks are timestamp-based [8]-[10]. For timestamp-based protocols, each newly arrived packet is assigned two timestamps, i.e., start and finish tags, based on (2). In [10], both tags are used to calculate a backoff value. The backoff value determines when the packet will be sent. The mechanism works as follows. To send a packet, the node monitors the channel until it is free, and then calculates a backoff value based on the two tags assigned to the packet. The backoff timer is incremented by one at each time slot until it reaches zero. If the node with a zero timer finds that the channel is free, the packet is transmitted. Nodes with zero-timer packets do not coordinate before the packets are transmitted. Thus, collisions may occur.

In [8] and [9], a flow graph must first be generated. Each vertex in the flow graph represents a flow. When two flows contend for resource, an edge is added between them. Depending on the scheduling discipline, one of the two tags is chosen as the service tag. The packet with the smallest service tag is sent first. The mechanisms in [8] and [9] differ in how they achieve spatial channel reuse. In [8], the graph coloring theory is used to mark the contending flows of a channel with different colors. The flows with the same color can be transmitted simultaneously. In [9], each packet of a flow is associated with a backoff value. This backoff value is equal to the number of flows contending for a channel but with smaller service tags. Once this backoff value reaches zero, the packet is transmitted.

The timestamp-based algorithms suffer two problems. One is the need to sort all packets in the queue in ascending order of their service tags. The other is that the virtual clock cannot be reinitialized to zero unless the system is empty [6].

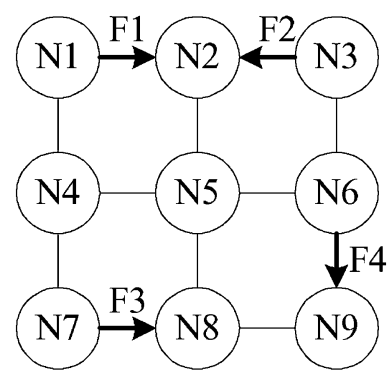

Fig. 1. Contending flows.

In [12], a credit-based fair scheduling algorithm is proposed for ad hoc networks. Each flow simply maintains a counter to record the transmission credit, instead of using two tags as in timestamp-based mechanisms. The basic scheduling concept is "the less excess in usage value, the higher the transmission priority." This approach makes use of clustering to implement spatial channel reuse.

2) Fair Scheduling With QoS Support: The mechanisms in [8] and [9] are two major timestamp-based solutions to provide fair scheduling for best effort flows in ad hoc networks. To extend a timestamp-based mechanism to support QoS, an intuitive approach is to assign each flow a different flow weight according to the respective service requirement. The higher the priority, the larger the flow weight. However, both mechanisms in [8] and [9] do not describe how flow weights are assigned. In the following, we extend [9] to enable QoS support with a distributed flow weight assignment scheme, and call the enhanced version of [9] as Q_EMLM-FQ in the rest of the paper.

The bottleneck considered fair scheduling (BCFS) mechanism [11] is extended from [8]. It assumes that a flow weight assignment scheme is given and introduces a new parameter called "contending power" to provide QoS service on top of the fair scheduling mechanism described in [8].

The mechanism in [12] is a credit-based mechanism for fair scheduling in ad hoc networks. In Section II, we extend [12] to provide QoS, and call the enhanced version of [12] CSAP in the rest of the paper. We then compare the performance of BCFS, Q_EMLM-FQ, and CSAP via simulation, and discuss the pros and cons of these three approaches.

a) Q_EMLM-FQ: Suppose that each QoS flow will specify its minimum bandwidth requirement for certain quality. For each flow $f$ passing through node $N$, the flow weight $w_{f}$ is defined as

$$
w_{f}=\operatorname{Resv}_{f}+\frac{1-\sum_{i \in B} \operatorname{Resv}_{i}}{\operatorname{Num}}
$$

where $\operatorname{Resv}_{f}$ is the minimum bandwidth requirement of flow $f$, Num is the total number of flows passing through node $N$, and $B$ is the set of backlogged flows. If flow $f$ is a best effort flow, its $\operatorname{Resv}_{f}$ value is zero. If flow $f$ is a guaranteed flow, its $\operatorname{Resv}_{f}$ is between zero and one. As such, guaranteed flows are assured to have flow weights larger than best-effort flows. Consequently, the service tags of guaranteed flows increase more slowly than best effort ones, allowing their service requirements to be satisfied. 
b) BCFS: To support QoS, a new parameter called "contending power" is defined in [11] as the scheduling metric for each flow. The contending power of flow $f$ is defined as follows. For each fully connected graph in which flow $f$ is involved, the flow weights of all flows in the graph are first summarized. The contending power of flow $f$ (denoted as $P(f)$ ) is equal to the maximum over the summation of the flow weights in each graph, say, $G_{f}$, involved by flow $f$, i.e.,

$$
P(f)=\max _{\forall G_{f}}\left\{\sum_{\forall f_{i} \in G_{f}} w_{f_{i}}\right\}
$$

where $f_{i}$ denotes a flow in graph $G_{f}$.

Fig. 2 illustrates how the contending power of each flow is calculated. Each vertex indicates a flow. $(F x, y)$ denotes the flow ID and its predefined flow weight, respectively. F1 is involved in two fully connected subgraphs, i.e., (F1, F2, F3) and (F1, F4, F5). Thus, F1 contends with F2 and F3, and also with F4 and F5. The total flow weights of these two subgraphs are $2+2+3=7$ and $2+4+4=10$, respectively. Therefore, the contending power of $\mathrm{F} 1$ is equal to $\max \{7,10\}=10$.

The scheduling rules of fair scheduling with QoS support in [11] are described as follows.

- The flow with the highest contending power has the priority to transmit packets.

- If there are multiple flows with the same contending power, the one with the least service tag has the priority to transmit.

- BCFS adopts the same graph-coloring approach as in [8] to implement spatial channel reuse. Each vertex is marked a color different from its direct neighbors. The set of flows in the same color can be transmitted simultaneously. The set of flows that has the maximum number of flows in the same color as the flow selected based on the first two rules is chosen to transmit simultaneously .

- If there are several sets with the same maximum number of flows that can be transmitted simultaneously, the set with the highest aggregate contending power is selected.

- BCFS sets a parameter called scheduling window to avoid starving flows that have low contending powers. A flow must stop transmission temporarily when a "scheduling window" worth of packets has been transmitted in a predefined time interval. In other words, based on a sliding window of size $\eta$, BCFS allows each flow to send up to $\eta$ packets in a predefined time interval.

BCFS suffers from the following problems.

- Flow weight assignment: As in [9], BCFS assigns each flow a positive integer as the flow weight, which indicates how the flow shares the resource. For example, if flows 0 , 1 , and 2 are assigned flow weights of 2,3 , and 1, respectively, the proportion of resource used by these three flows is 2:3:1. However, there is no word in [11] to mention how flow weights are assigned and learned and to discuss the impact of different flow weight assignments on the performance of BCFS.

- The impact of flow topology: In BCFS, the contending power of each flow is calculated for scheduling. The flow

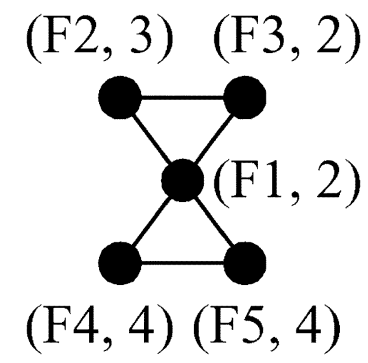

Fig. 2. An example of contending power.

with the highest contending power has the priority to transmit. However, a flow with a higher contending power does not imply a higher flow weight. Recall that the flow weight is used to indicate how the flow shares system resource. The higher the flow weight, the more time slots the flow can use. For BCFS, the contending power of a flow is determined based on its neighbors in the flow graph. Both the node degree and neighbor flows' weights affect the contending power of the flow. In addition, the spatial reuse mechanism adopted may further reverse the service priority. As a result, a flow with a lower weight may be allocated more slots than one with a higher flow weight.

Fig. 3 illustrates why the performance of BCFS is affected by flow topology. Each vertex represents a flow. Let $x, y$, and $z$ in $x(y, z)$ denote flow ID, flow weight, and contending power, respectively. In this example, flow 5 has both the highest flow weight and the highest contending power. Thus, it has the highest priority to transmit packets. However, flow 4, which has the second largest flow weight, may not have the second highest priority to transmit packets. The reason is described as follows. Considering spatial channel reuse, flow 5 can be transmitted simultaneously with some other flows. For example, it can be transmitted with flows 1,6 , and 7, or with flows 1 and 4 . Flow 5 will choose the first set (i.e., flows $1,6,7)$ because the first set has more flows (i.e., 1 , 6 , and 7) to be transmitted simultaneously. As a result, flow 4 may be allocated fewer slots than flows 1 or 7 , even though flow 4 has a higher weight than either flow. The example above shows that the flow topology significantly affects the performance of BCFS. In [11], however, there is no discussion on this issue.

- A centralized scheduling mechanism: In [11], a globally centralized coordinator is assumed available to schedule packet transmissions, but there is no description of how such a centralized mechanism is implemented in a fully distributed ad hoc network.

\section{Problem Specification for CSAP}

In this paper, we propose a new mechanism called the creditbased slot allocation protocol to provide fair scheduling with QoS support for multimedia ad hoc networks. We consider a mix of best effort packets and guaranteed flows, and use "bandwidth" as the QoS metric due to its importance for real-time applications. In [6], bandwidth requirement in time-slotted network is measured in terms of fraction of a time slot per unit time, 


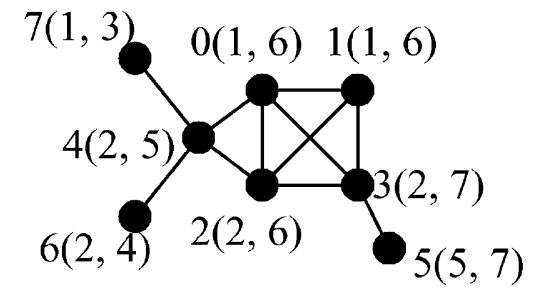

Fig. 3. An illustrative example for BCFS.

where unit time is a time slot. As in [6], we use a fraction of one slot time to denote the bandwidth requirement of each guaranteed flow.

The objectives of CSAP are described as follows.

1) Avoid collisions caused by simultaneous, uncoordinated transmissions, thus improving system throughput.

2) Maximize spatial channel reuse, thus maximizing global bandwidth utilization.

3) Guarantee minimum bandwidth for guaranteed flows, and provide fair share of residual bandwidth for all flows (i.e., both best effort and guaranteed flows). Let $F_{b}$ and $F_{g}$ denote the sets of best effort and guaranteed flows in a cluster, respectively. The objective of CSAP is to make $E_{1}$ and $E_{2}$ in (5) and (6) as close to zero as possible. Both flows $i$ and $j$ are in $F_{g}$ and have minimum bandwidth requirements $r_{i}$ and $r_{j}$, respectively. Flow $k$ is in $F_{b} . W_{i}\left(t_{1}, t_{2}\right), W_{j}\left(t_{1}, t_{2}\right)$, and $W_{k}\left(t_{1}, t_{2}\right)$ denote the aggregate resource received by flows $i, j$, and $k$, respectively, in time interval $\left[t_{1}, t_{2}\right] . C_{i}\left(t_{1}, t_{2}\right)$ is the cumulated credit of flow $i$ in $\left[t_{1}, t_{2}\right] . S_{i}\left(t_{1}, t_{2}\right)$ and $S_{j}\left(t_{1}, t_{2}\right)$ are the residual bandwidth shared by flows $i$ and $j$ in $\left[t_{1}, t_{2}\right]$, respectively

$$
\begin{aligned}
& E_{1}=\frac{W_{i}\left(t_{1}, t_{2}\right)-S_{i}\left(t_{1}, t_{2}\right)}{r_{i}}-\frac{W_{j}\left(t_{1}, t_{2}\right)-S_{j}\left(t_{1}, t_{2}\right)}{r_{j}} \\
& E_{2}=\left|W_{i}\left(t_{1}, t_{2}\right)-C_{i}\left(t_{1}, t_{2}\right)-W_{k}\left(t_{1}, t_{2}\right)\right| .
\end{aligned}
$$

The rest of this paper is organized as follows. Section II describes the credit-based slot allocation protocol for fair scheduling with QoS support. Section III shows the simulation results. The conclusion is drawn in Section IV.

\section{CRedit-Based Slot Allocation Protocol (CSAP)}

This section describes the proposed credit-based slot allocation protocol in details. The characteristics of CSAP are described as follows.

1) A two-tier hierarchy is used to avoid uncoordinated transmissions.

2) A cluster-based mechanism is used to maximize spatial channel reuse.

3) A credit-based mechanism is used to provide QoS service for guaranteed flows while ensuring fair scheduling.

Note that our mechanism works with different residual bandwidth allocation schemes, including fair scheduling for best effort flows only, for a mix of best effort and guaranteed flows, or for a mix of flows but with an upper limit on the resource share for guaranteed flows. In this paper, we just demonstrate the second case, i.e., for a mix of flows without an upper bound on each flow.

\section{A. Assumptions}

1) Wireless media may exhibit time-varying errors, which affect network users in a number of ways, such as fading, interference, link errors, and collisions. In this paper, we only consider the errors caused by collisions.

2) We consider packet-switched multihop wireless networks, but do not consider host mobility as in all related work in ad hoc networks [8]-[12].

3) We assume a time-division multiple-access (TDMA)based system on a single channel shared by all hosts. To avoid collisions, code-division multiple access can be overlaid on the top of the TDMA system. We assume a code assignment algorithm is running in the lower layer of our system as in [1].

4) There are always some flows backlogged.

\section{B. Service Registration}

1) Cluster-Based Mechanism: CSAP is a cluster-based mechanism. The network is logically partitioned into clusters. Each cluster has exactly one scheduler. Clusters may be overlapped, each with a designated code to avoid interferences. CSAP can cooperate with any existing clustering algorithm (e.g., [13] and [14]) to form clusters. For example, a cluster could be formed as follows. Each mobile node periodically broadcasts beacons to nodes located within its transmission range. Based on the received beacons, mobile nodes learn of their neighbors and related information, such as node ID, node stability, etc., for operation. According to the selected criterion, one node is elected as the scheduler per cluster. Each scheduler periodically advertises itself to its neighbors, from which newly arriving mobile nodes learn where to register. Those nodes that can hear the same scheduler form a logical cluster. Each node can hear several schedulers, but can only be registered with one scheduler at a time.

2) Table Structure: CSAP defines two scheduling tables.

a) Node allocation table (NAT): Maintained by the scheduler; each entry of the NAT is for a (node, service type) pair and is of six fields: a node ID, a service type, a Resv, a Credit, a Usage, and an Excess. For a guaranteed service entry, the value in the Resv field indicates the total reservation level required to serve all guaranteed flows for the corresponding node. For a best effort entry, the Resv value represents the total number of best effort flows scheduled by the node.

b) Flow allocation table (FAT): Maintained by the node; each entry of the FAT is for a flow and is of seven fields: a flow ID, a scheduler ID, a service type, a Resv, a Credit, a Usage, and an Excess.

3) Path Construction: Before a transmission starts, the sender of the flow must first determine a flow path, irrespective of whether it is a best effort or a guaranteed (i.e., QoS) flow. Best effort flows do not place any resource requirement on the nodes along the path. However, for a QoS flow, each node along the path should provide resource for the flow to meet its minimum requirement. CSAP can be easily integrated with any of the existing work on ad hoc routing for path construction (e.g., [1]-[15]). For best effort flows, ad hoc routing is used; for guaranteed flows, QoS routing is required. Below we just 
simply describe an existing path construction mechanism, and illustrate how CSAP works based on the mechanism in the rest of this paper.

To construct a path, a sender broadcasts an RREQ message to its neighbors. Each node receiving this RREQ will enter the service registration phase described in the next section. If the registration fails, this RREQ message will be discarded. Duplicated RREQ messages are discarded when received. When an RREQ message reaches the destination, the destination will send an RREP message in reply along the reverse path back to the sender. Unlike the RREQ message, the RREP message is delivered via unicast. Upon receiving an RREP message at a node, the reservation is confirmed. This process continues until the RREP message reaches the sender, when the path construction phase terminates.

4) Service Registration: Upon receipt of an RREQ message, a node checks if the following condition holds, namely, the summation of the total reserved usage (denoted as Resv) of all flows scheduled by the node, including the newly arriving one, is less than or equal to the target link utilization. If the condition holds, the node further sends a resource Allocation ReQuest (ARQ) to its scheduler to check if the condition holds at the scheduler as well. If the condition fails to hold at the node or the scheduler, the request is denied and the RREQ packet is discarded; otherwise, the request is tentatively accepted. The scheduler creates a temporary entry for the node in the NAT, and responds to the node with a temporary acceptance. The node in turn creates a temporary entry for the flow in the FAT, and then rebroadcasts this RREQ to its neighbors. Note that temporary entries in the scheduling table (i.e., in both FAT and NAT) are not involved in the scheduling process.

Once the corresponding RREP message is received, the node will change the entry in the FAT from temporary to active, and will inform its scheduler to make the entry in the NAT active. Those temporary entries not confirmed by the corresponding RREP within a predefined period of time will be cleared in the scheduling table.

\section{Two-Tier Slot Allocation}

1) Scheduling Parameters: There are four parameters defined in CSAP:

a) Resv: A fraction of one time slot, used to indicate the minimum requirement of a flow. For a guaranteed flow, the Resv value is a positive real number between zero and one. For a best effort flow, its Resv value is always zero.

b) Credit: The cumulative time slots reserved for a guaranteed flow to meet the QoS demand, or the remaining slot quota for a best effort flow per iteration. ${ }^{1}$ For the guaranteed service type, the value of an increase at each time slot is equal to a Resv value. For the best effort service type, the Credit value is initialized to the total number of best effort flows currently scheduled by the node, and is decreased by one after a slot is assigned. Note that for a best effort flow, the Credit field is only valid in the NAT and is always set to zero in FAT.

\footnotetext{
${ }^{1}$ Each iteration indicates a cycle in which the slot quota is counted down from
} the original value to zero. c) Usage: The cumulative time slots assigned to a request. For a scheduler, it records a Usage for each node. For a node, it records a Usage for each flow. The Usage value can be a zero or any positive integer.

d) Excess: This value is used to determine to whom the next time slot is assigned. The next slot is assigned to the request with the smallest Excess value. For guaranteed-service entries, the Excess values are equal to "Usage minus Credit" and are updated at each time slot. For best effort service entries, the Excess values are initialized to zero, and incremented by one at each "update." The Excess value of a best effort service entry is only updated when the corresponding Credit value is count down to zero. Once the Excess value has been updated, the Credit value is reset to a Resv value, and a new iteration starts.

2) Slot Allocation: The slot allocation is based on a two-tier mechanism. The scheduler assigns time slots based on the first tier slot allocation mechanism. The node, say, N, to whom the time slot is assigned then in turn assigns the time slot to a flow based on the second tier slot allocation mechanism.

Suppose that there are $m$ and $n$ entries in NAT and FAT, respectively. The $m$ entries in NAT can be divided into two sets: $S_{g}$ and $S_{b}$, which indicate guaranteed and best effort service types, respectively. The first tier slot allocation mechanism works as follows.

a) The scheduler assigns the next time slot to the node with the smallest Excess value in the NAT at the decision time.

Suppose that node A is the node scheduled to send at the next time slot. The scheduler then updates the values of Credit, Usage, and Excess in all entries of the NAT, as follows.

b) Increment the Usage value of the entry for node A and leave all the others intact, i.e., Usage $(A) \leftarrow$ Usage $(A)+1$, where $\operatorname{Excess}(A)=\min \{\operatorname{Excess}(x) \mid x=$ $1,2, \ldots, m\}$.

c) Increase Credit by Resv for each guaranteed-service entry in the NAT, i.e., Credit $(x) \leftarrow \operatorname{Credit}(x)+$ $\operatorname{Resv}(x), \forall x \in S_{g}$. If the time slot is assigned to a best effort service entry, the Credit value of node $A$ is decremented by one, i.e., $\operatorname{Credit}(A) \leftarrow \operatorname{Credit}(A)-1$.

d) Update the Excess values of all guaranteed-service entries in the NAT, i.e., $\operatorname{Excess}(x) \leftarrow \operatorname{Usage}(x)-$ $\operatorname{Credit}(x), \forall x \in S_{g}$. If the time slot is assigned to a best effort service entry and the Credit value of node A equals zero, the Excess value is incremented by one and the Credit value is reset to the Resv value, i.e., if $\operatorname{Credit}(A)=0, \operatorname{Excess}(A) \leftarrow \operatorname{Excess}(A)+1$, and $\operatorname{Credit}(A) \leftarrow \operatorname{Resv}(A)$; the Excess values of other best effort entries stay unchanged.

The second tier slot allocation algorithm works as follows.

a) The node cannot schedule any flow for transmission unless a time slot is assigned to it. Once a time slot is assigned, the node assigns the time slot to the flow that satisfies the following two conditions: i) the flow has the smallest Excess value and ii) the flow's service type matches the service type of the node granted by the scheduler. 
Suppose that flow $F$ is scheduled to transmit at the next time slot. The node updates the values of Credit, Usage, and Excess in all entries of the FAT, as follows.

b) Increment the Usage value of the entry for flow $F$, and leave the others intact. Usage $(F) \leftarrow \operatorname{Usage}(F)+1$, where $\operatorname{Excess}(F)=\min \{\operatorname{Excess}(y) \mid y=1,2, \ldots, n\}$.

c) Update the Excess values of all entries in the FAT, i.e., $\operatorname{Excess}(y) \leftarrow \operatorname{Usage}(y)-\operatorname{Credit}(y), y=1,2, \ldots, n$.

3) $\operatorname{Credit}(y)=\operatorname{Credit}(y)+\operatorname{Resv}(y), y=1,2, \ldots, n$.

\section{Global Fairness in CSAP}

So far, we have described how to ensure per-cluster fairness while providing QoS service for guaranteed flows. In [8], [9], and [11], the mechanisms focus on global fairness. That is, no matter where the flow is, the resource usage is proportion to the flow weight. To achieve this goal, they set a parameter called scheduling window to ensure global fairness. CSAP can be easily extended to ensure global fairness when a scheduling window mechanism similar to that described in [8], [9], and [11] is implemented. The window mechanism in [8], [9], and [11], however, has some problem when applied directly to CSAP. For example, the service guarantee fails if the timer expires before the time slots for the minimum requirement of a flow are allocated.

Let $\eta$ be the scheduling window for CSAP. The scheduling rules for global fairness in CSAP are revised as follows.

1) When a flow, say, $i$ has sent up to $\eta$ additional packets in a period of time after its minimum QoS requirement has been satisfied, the node, say, $n$ will stop scheduling further time slots to flow $i$, and will send an update message to the scheduler to temporarily degrade its Resv level. For example, node $\mathrm{N}$ manages three flows $\mathrm{F} 0, \mathrm{~F} 1$, and F2, with a Resv of $0.2,0.2$, and 0.3 , respectively. All these three flows are registered with the same scheduler S. Suppose F1 has sent up to $\eta$ packets in a period of time. Thus, node $\mathrm{N}$ will send an update message to $\mathrm{S}$ to degrade its total Resv level from 0.7 to 0.5 .

2) For a scheduler, the rules to make scheduling decisions are intact as described in Section II-C2.

3) When node $\mathrm{N}$ has been assigned the next time slot, it will in turn assign the slot to the flow satisfying the following three conditions: i) the flow is not frozen to be scheduled, ii) the flow's service type matches the service type of the node granted by the scheduler, and iii) the flow has the smallest Excess value.

After the expiry of the predefined period of time, node $\mathrm{N}$ resumes the scheduling for frozen flows and informs the scheduler to resume their original Resv levels.

\section{SimULATION}

In this section, the performance of CSAP, Q_EMLM-FQ, and BCFS is compared by simulation. There are 20 mobile nodes randomly distributed in a 670 by 670 area. We randomly select nodes, some as flow senders and some as flow receivers. Each flow may be of best effort or guaranteed. For best effort flows, their Resv values are set to zeros, and for guaranteed flows, the values are randomly generated between zero and one. The packet length is fixed and is assumed to occupy one time slot. The time period and the window size used for the scheduling window of the global fairness mechanism are set to 200 time slots and 150 packets, respectively.

\section{A. Performance Metrics}

1) Share Degree $(\phi)$ : Share degree $(\phi)$ is the percentage of residual bandwidth shared by each flow. Here the residual bandwidth means the bandwidth left after the minimum requirements requested by all QoS flows have been allocated. The share degree of flow $\mathrm{F}$ is Usage $(F)$ divided by total number of time slots and then minus $\operatorname{Resv}(F)$, i.e., $\phi=(\operatorname{Usage}(F) /$ total slots $)-$ $\operatorname{Resv}(F)$. The share degree of a flow is value between $[-1,1]$. The larger the value, the more residual bandwidth the flow has used. A flow with a negative $\phi$ value must be a QoS flow whose minimum bandwidth requirement is not satisfied.

2) Satisfaction Index $(\rho)$ : This parameter is used to indicate how well all QoS flows are satisfied by each approach. $\rho$ is defined in a way similar to the definition of the fairness index in [16]. The parameter $x_{i}$ indicates whether or not a QoS flow has been satisfied. If the time slot usage of a QoS flow is larger than or equal to its minimum requirement, $x_{i}=1$; otherwise, $x_{i}$ indicates the insufficient portion. Thus $\rho=(1 / n) \sum_{i=1}^{n}\left(x_{i} / x_{f}\right)$, where

$$
x_{i}= \begin{cases}1, & \text { if } \phi(i) \geq 0 \\ 1-\alpha \times \frac{|\phi(i)|}{\operatorname{Resv}(i)}, & \text { if } \phi(i)<0\end{cases}
$$

whose $\alpha \geq 1$ is a weighting factor, and $x_{f}=$ $\left(\sum_{i=1}^{n} x_{i}^{2}\right) /\left(\sum_{i=1}^{n} x_{i}\right)$. The satisfaction index for an approach is a value between zero and one. The larger the value, the more flows are satisfied with the desired service provided by the approach.

3) Fairness Index $(\kappa)$ : This parameter indicates how fair the residual bandwidth is shared by all flows for each approach. The parameter is defined as

$$
\kappa=\frac{1}{m}\left\{\sum_{j=1}^{m}\left(\frac{1}{n_{j}} \sum_{k=1}^{n_{j}} \frac{x_{k}}{x_{f}(j)}\right)\right\}
$$

where $m$ is the number of clusters, $n_{j}$ is the number of flows in cluster $j ; x_{k}$ indicates the share degree of flow $k$, and

$$
x_{k}= \begin{cases}\phi(k), & \text { if } \phi(k) \geq 0 \\ 0, & \text { if } \phi(k)<0 .\end{cases}
$$

The $x_{f}$ value of cluster $j$ is $x_{f}(j)=\left(\sum_{k=1}^{n_{j}} x_{k}^{2}\right) /\left(\sum_{k=1}^{n_{j}} x_{k}\right)$. Fairness index $\kappa$ is a value between zero and one. The larger the value, the fairer the residual bandwidth is shared among all flows. Note that this index can be used for global fairness when $m$ is set to one.

4) Network Throughput: Network throughput is defined as $\sum_{i \in N}(\operatorname{Usage}(i) /$ total slots $)$, where $N$ is the set of all flows in the ad hoc network. The larger the network throughput, the more efficient the spatial channel reuse.

\section{B. Simulation Results}

In the following, simulation results are presented. We first examine the single cluster case, and then multiple clusters. We 
also demonstrate that CSAP can still ensure minimum bandwidth requirements for QoS flows and provide global fairness for all flows in the last simulation. Finally, we show the impact of different clustering schemes and cluster size on the performance of each mechanism.

1) Single Cluster: We first present the simulation results for single cluster scenarios. The transmission range of each node is set to 670 units. In the first experiment, five pairs of nodes in a single cluster are randomly selected as flow senders and receivers. Five flows are randomly generated: two guaranteed flows and three best effort flows. Flows 0 and 1 are guaranteed flows with a Resv value of 0.58 and 0.02 , respectively. Flows 2,3 , and 4 are effort flows. Ideally, the share of the residual bandwidth for each flow should be $(1-0.58-0.02) / 5=0.08$.

Fig. 4(a) shows the network topology and flow distribution. In this figure, the square node indicates the cluster scheduler, the circles represent mobile nodes, an arrow shows the direction of a flow from the source to the destination, the solid lines indicate best effort flows, and the dotted lines indicate guaranteed flows. Fig. 4(b) shows the corresponding flow graph as described in Section I-C2b. $x$ and $y$ in $x(y)$ means the flow ID and the contending power of the flow, respectively. The flow graph is a four-degree fully connected graph, in which each flow has four neighbors and has a contending power of five. Since each flow has the same value of contending power, the scheduling decision made by BCFS is based on the least service tag. Thus, it has the same result as Q_EMLM-FQ. Fig. 4(c) shows the flow throughputs of the five flows for each mechanism. We see that CSAP meets the minimum requirements of all flows [see flows 1 and 2 in Fig. 4(c)], and thus has a satisfaction index of one [Fig. 4(d)]. In addition, it has an equal share of residual bandwidth among five flows, each with a share degree of 0.08 , the same as the ideal sharing [Fig. 4(e)]. Thus, CSAP has a fairness index of one [Fig. 4(f)].

Both Q_EMLM-FQ and BCFS allocate resource equally to the five flows [Fig. 4(c)], despite different types of service requested by the flows. As a result, they do not guarantee the minimum bandwidth requirement of the guaranteed flow [i.e., flow 0 in Fig. 4(c)] and has a lower satisfaction index. Meanwhile, both Q_EMLM-FQ and BCFS have a flow with a negative share degree. Thus, they have a lower fairness index, which is 0.7984 .

We then randomly generate ten guaranteed flows in a single cluster ad hoc network. Fig. 5(a) shows the network topology and Fig. 5(b) shows the flow information. Fig. 5(c) shows the flow throughput of the ten flows for each mechanism. Both Q_EMLM-FQ and BCFS allocate slots relatively equally to each flow, irrespective of whether it is a best effort or a guaranteed flow. Three guaranteed flows are not satisfied [i.e., flows 3, 6, 8 in Fig. 5(c)]. Thus, both have a satisfaction index of 0.8934 [Fig. 5(d)]. In addition, they do not share the residual bandwidth fairly [see Fig. 5(e)], and have a fairness index of 0.4314 [Fig. 5(f)]. CSAP satisfies the requirements of all flows except flow 6 , and shares residual bandwidth more fairly. Thus, it has a higher satisfaction index and a higher fairness index.

2) Multiple Cluster: We then extend the experiment from one cluster to multiple clusters. We randomly generate a mix of best effort and guaranteed flows. This time the network is logically partitioned into four clusters. Flows $0,1,3,6$, and 7
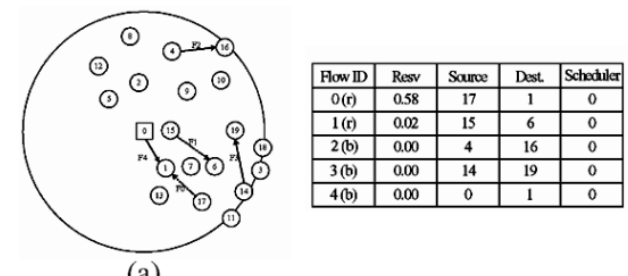

(a)

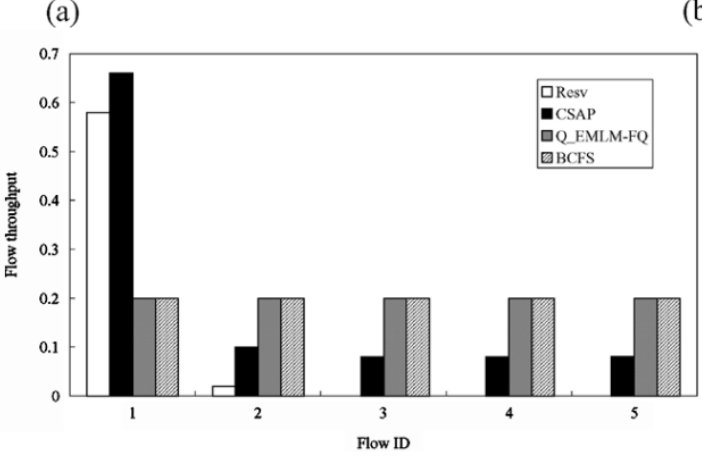

(c)

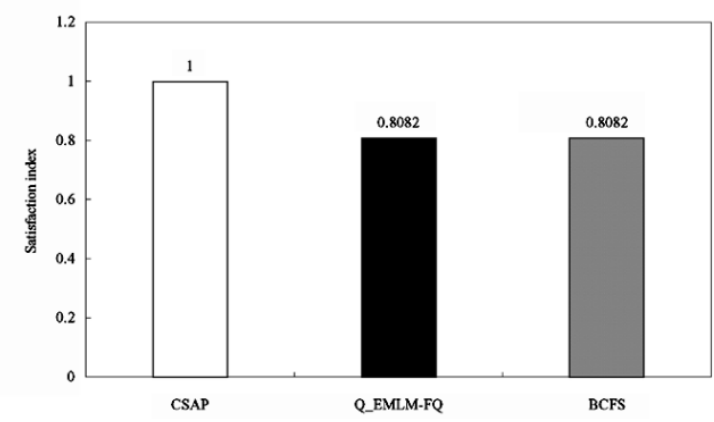

(d)

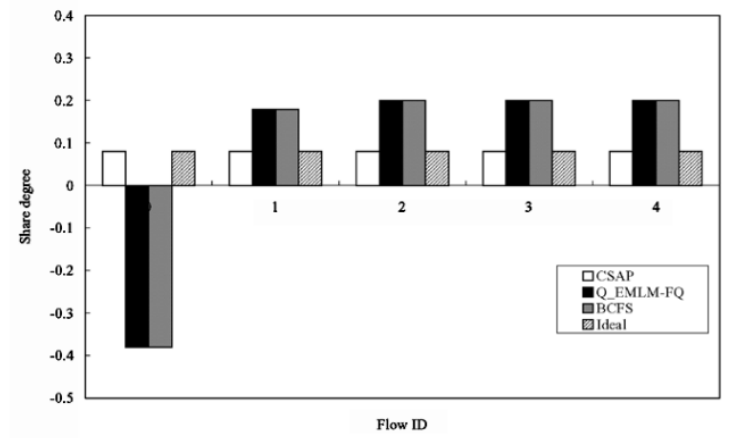

(e)

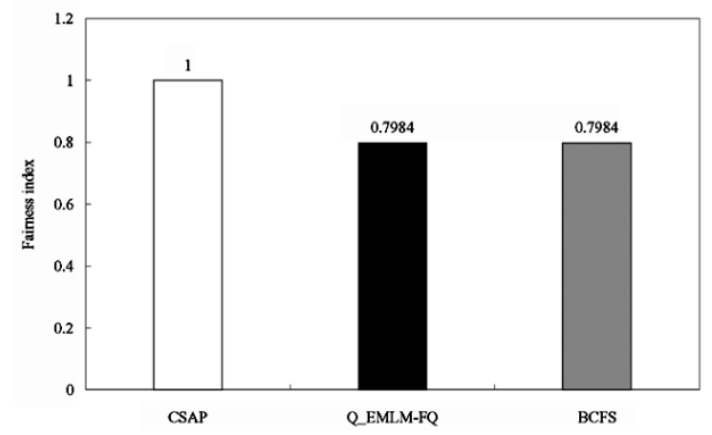

(f)

Fig. 4. Single cluster with a mix of flows. (a) Network topology and flow information, (b) flow graph, (c) flow throughput, (d) satisfaction index, (e) share degree, and (f) fairness index.

are scheduled by node 0 . Ideally, each should share a fraction $(1-0.1-0.27-0.05-0.26) / 5=0.064$ of the residual 


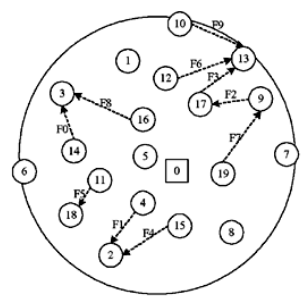

(a)

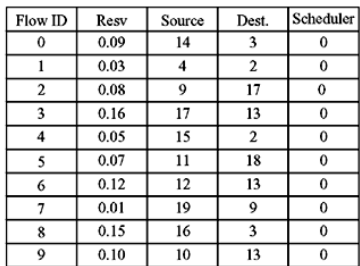

(b)

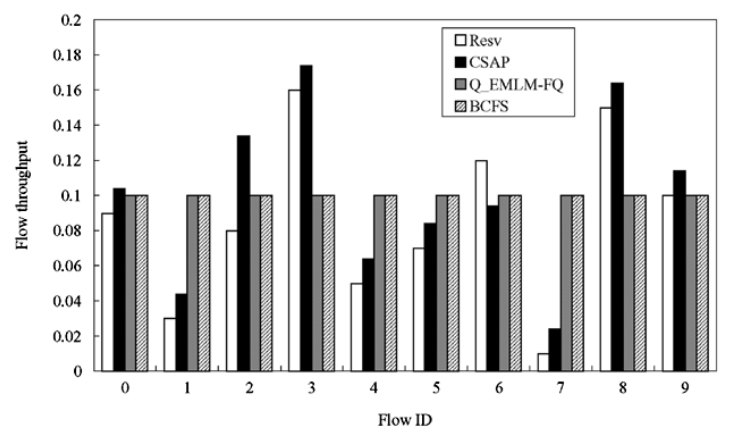

(c)

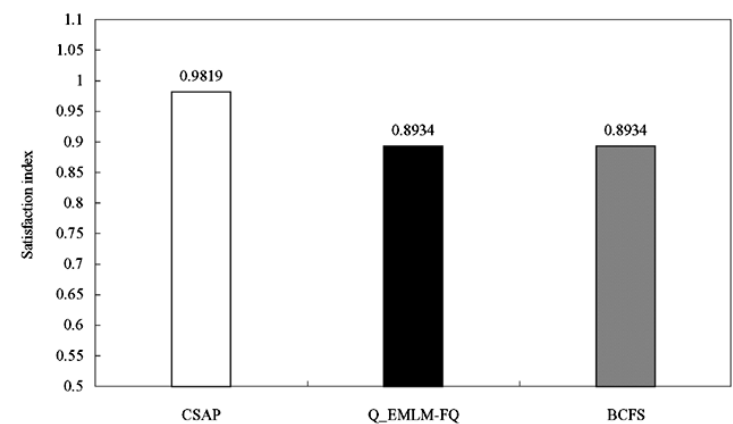

(d)

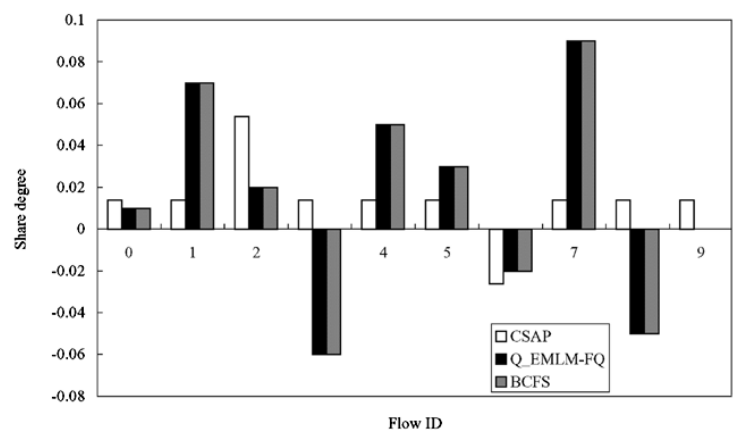

(e)

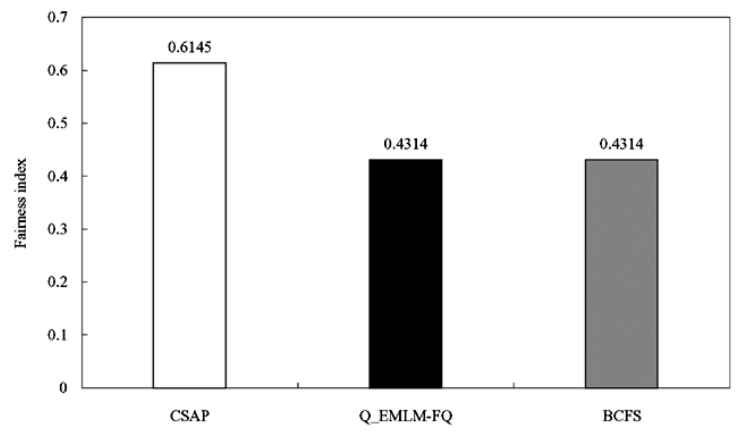

(f)

Fig. 5. Single cluster with guaranteed flows only. (a) Network topology, (b) flow information, (c) flow throughput, (d) satisfaction index, (e) share degree, and (f) fairness index.

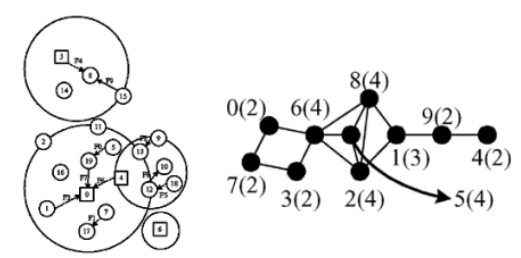

(a)

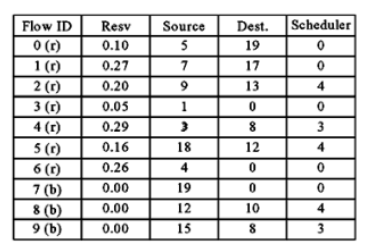

(b)

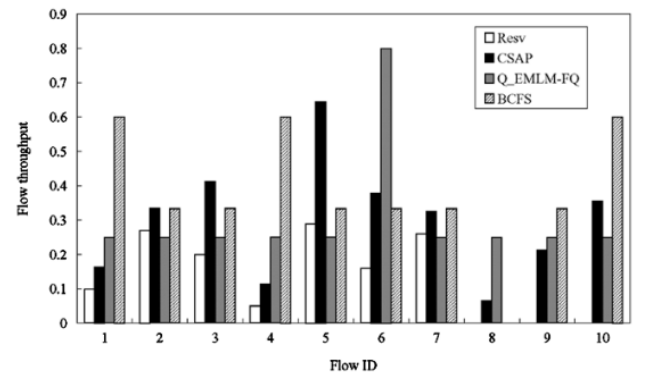

(c)

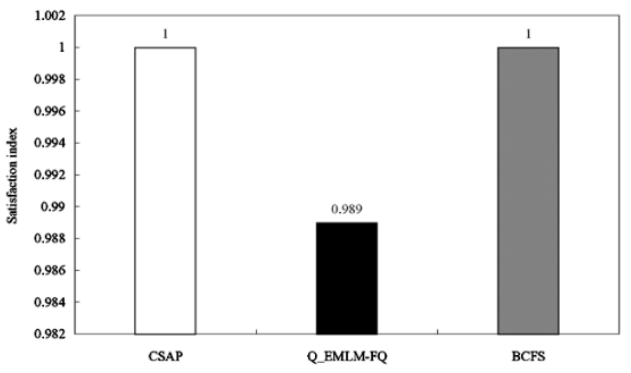

(d)

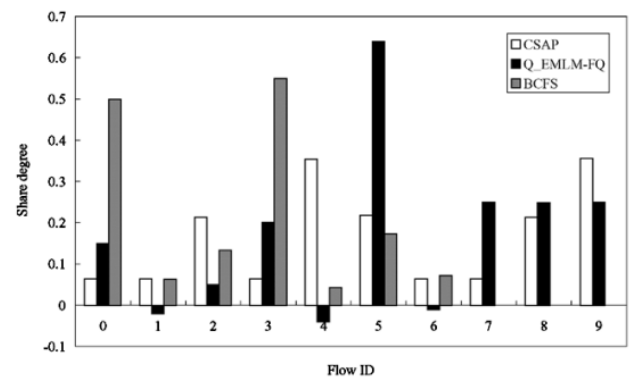

(e)

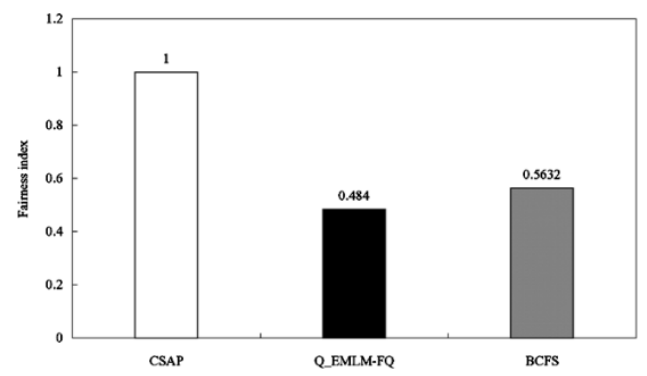

(f)

Fig. 6. Multiple clusters with a mix of flows. (a) Network topology, (b) flow information, (c) flow throughput, (d) satisfaction index, (e) share degree, and (f) fairness index.

bandwidth. Flows 2,5 , and 8 are managed by node 4 , and each should have an equal share of $(1-0.20-0.16) / 3=0.2133$. Both flows 4 and 9 are managed by node 3 , and each shares a fraction of residual bandwidth equal to $(1-0.29) / 2=0.355$. Fig. 6(a) shows the network topology, the flow distribution, and the flow graph. The detailed flow information is shown in Fig. 6(b). Fig. 6(c) and (e) shows the flow throughput and 


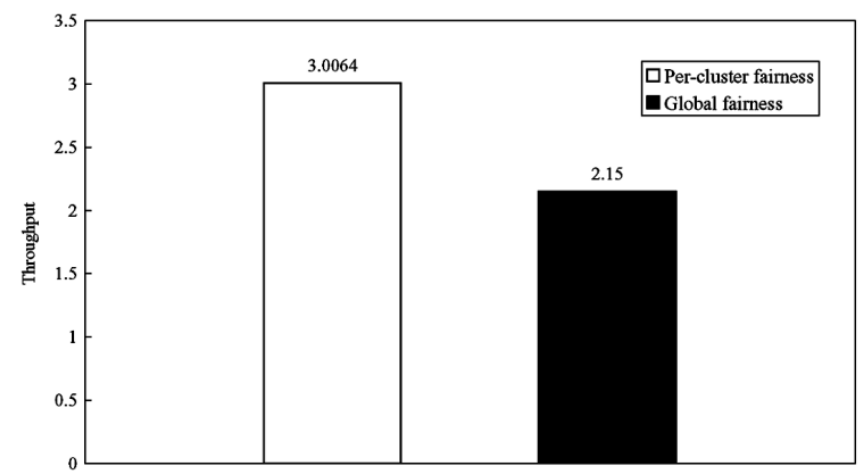

(a)

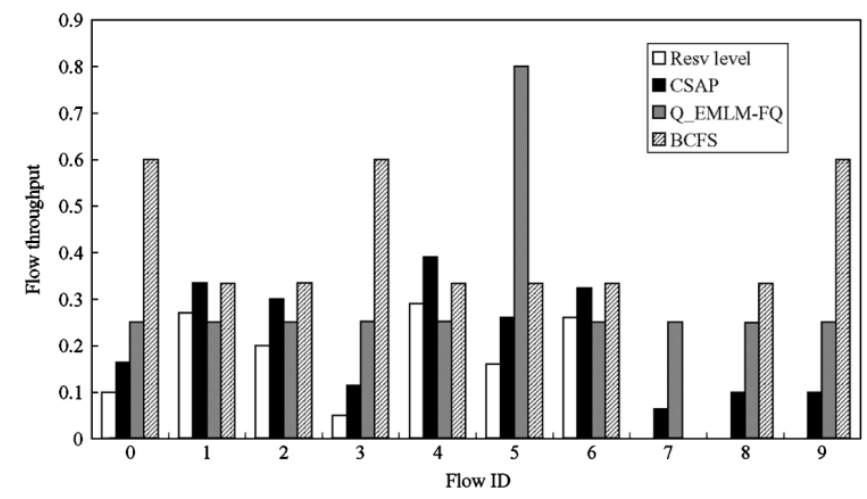

(b)

Fig. 7. Global fairness. (a) Per-cluster versus global fairness and (b) flow throughput with global fairness.

the share degree of each flow. Fig. 6(d) and (f) shows the satisfaction and fairness indexes of each approach.

Again, CSAP provides the minimum bandwidth requirements for all flows and ensures fair share for per-cluster residual bandwidth. As a consequence, both the satisfaction and fairness indexes are equal to one. Q_EMLM-FQ, on the other hand, cannot always satisfy the requirements of all flows. BCFS can meet the minimum requirements of all QoS flows, but fails to fairly share the residual bandwidth.

The last experiment is conducted to observe the impact of global fairness on the performance of CSAP. Fig. 7(a) shows the average system throughputs for per-cluster and global fairness in the multicluster scenario shown in Fig. 6. We see that the throughput of CSAP degrades when the global fairness mechanism described in Section II-D is implemented. Fig. 7(b) shows that CSAP with the global fairness mechanism still ensures the minimum requirements of QoS flows and provides global fairness for all flows in sharing the residual bandwidth.

\section{CONCLUSION}

In this paper, we have proposed a credit-based mechanism called CSAP to provide fair scheduling with QoS supports for multimedia wireless ad hoc networks. In addition, we have proposed Q_EMLM-FQ, which introduces a distributed flow weight calculation scheme to [9], to make it capable of QoS support. We have also pointed out some problems of BCFS, which is extended from [8] for QoS service.
To compare CSAP with Q_EMLM-FQ and BCFS quantitatively, some performance indexes are defined. We have also conducted simulations to observe the fairness and satisfaction degrees of these three approaches. The results show that of these three mechanisms, CSAP performs the best because it can guarantee QoS flows with the minimum bandwidth requirements, and share the residual bandwidth more fairly with all flows in the ad hoc network. Both Q_EMLM-FQ and BCFS usually allocate bandwidth equally, despite different types of service requested by the flows. As a result, they may not function very well.

\section{REFERENCES}

[1] C. R. Lin and J.-S. Liu, "QoS routing in ad hoc wireless networks," IEEE J. Select. Areas Commun., vol. 17, pp. 1426-1438, Aug. 1999.

[2] S. Chen and K. Nahrstedt, "Distributed quality-of-service routing in de hoc networks," IEEE J. Select. Areas Commun., vol. 17, pp. 1488-1505, Aug. 1999

[3] P. Gevros, J. Crowcroft, P. Kirstein, and S. Bhatti, "Congestion control mechanisms and the best effort service model," IEEE Network, pp. 16-26, May/June 2001.

[4] P. Goyal, H. M. Vin, and H. Cheng, "Start-time fair queueing: A scheduling algorithm for integrated services packet switching networks," IEEE/ACM Trans. Networking, vol. 5, pp. 690-704, Oct. 1997.

[5] J. M. Jaffe, "Bottleneck flow control," IEEE Trans. Commun., vol COM-29, pp. 954-962, July 1981.

[6] E. Hossain and V. K. Bhargava, "A centralized TDMA-based scheme for fair bandwidth allocation in wireless IP networks," IEEE J. Select. Areas Commun., vol. 19, pp. 2201-2214, Nov. 2001.

[7] Y. Cao and V. O. K. Li, "Scheduling algorithms for broadband wireless networks," Proc. IEEE, vol. 89, pp. 76-87, Jan. 2001.

[8] H. Luo and S. Lu, "A topology-independent fair queueing model in ad hoc wireless networks," in Proc. IEEE Int. Conf. Network Protocols, Nov. 2000, pp. 325-335.

[9] — "A self-coordinating approach to distributed fair queueing in ad hoc wireless networks," in Proc. IEEE INFOCOM, Apr. 2001, pp. 1370-1379.

[10] N. H. Vaidya and P. Bahl, "Fair scheduling in broadcast environments,", Microsoft Research Tech. Rep. MSR-TR-99-61.

[11] X. Wu, Y. Gao, H. Wu, and B. Li, "Fair scheduling with bottleneck consideration in wireless ad-hoc networks," in Proc. IEEE Int. Conf. Computer Communication and Networks, Oct. 2001, pp. 568-572.

[12] H. L. Chao and W. Liao, "Credit-based fair scheduling in wireless ad hoc networks," in Proc. IEEE Vehicular Technology Conf., Sept. 2002.

[13] H.-C. Lin and Y.-H. Chu, "A clustering technique for large multihop mobile wireless networks," in Proc. IEEE Vehicular Technology Conf., Sept. 2000, pp. 1545-1549.

[14] D. J. Baker and A. Ephremides, "The architecture organization of a mobile radio netework via distributed algorithm," IEEE Trans. Commun., vol. COM-29, pp. 1694-1701, Nov. 1981.

[15] C. E. Perkins and E. M. Royer, "Ad-hoc on demand distance vector routing," in Proc. 2nd IEEE Workshop Mobile Computing Systems and Applications, Feb. 1999, pp. 90-100.

[16] R. K. Jain, D. W. Chiu, and W. R. Hawe, "A quantitative measure of fairness and discrimination for resource allocation in shared computer system," DEC Tech. Rep. DEC-TR-301, 1984.

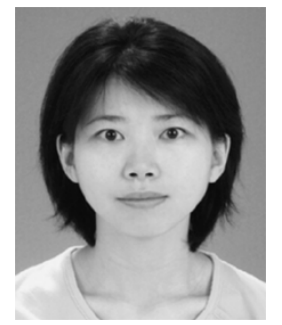

Hsi-Lu Chao (S’00) received the B.S. degree in electronic engineering from Fengchia University, China, in 1992 and the M.S. degree in electrical engineering from University of Southern California, Los Angeles, in 1996. She is currently pursuing the Ph.D. degree in the Department of Electrical Engineering, National Taiwan University, Taipei, Taiwan, R.O.C.

Her research interests include wireless communication and QoS issues in ad hoc networks. 


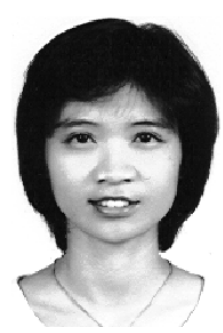

Wanjiun Liao (M'97) received the B.S. and M.S. degrees from National Chiao Tung University, Taiwan, R.O.C., in 1990 and 1992, respectively, and the Ph.D. degree in electrical engineering from the University of Southern California, Los Angeles, in 1997.

She joined the Department of Electrical Engineering, National Taiwan University (NTU), Taipei, Taiwan, as an Assistant Professor in 1997. Since August 2000, she has been an Associate Professor. Her research interests include wireless networks, optical networks, and broadband Internet. She is actively involved in the international research community.

Dr. Liao is an Associate Editor for IEEE TRANSACTIONS ON WIRELESS COMMUNICATIONS. She has received many research awards, including the Outstanding Research Paper Award in Electrical Engineering from the University of Southern California in 1997. Two papers she co-authored with her students received the Best Student Paper Award at the First IEEE International Conferences on Multimedia and Expo in 2000 and the Best Paper Award in the First IEEE International Conference on Communication, Circuits, and Systems in 2002. She was elected as one of Ten Distinguished Young Women in Taiwan in 2000 and is listed in Marquis Who's Who in 2001-2003 and the Contemporary Who's Who in 2003. 